\title{
Relationship between Oxidative Stress, Inflammation and Dyslipidemia with Fatty Liver Index in Patients with Type 2 Diabetes Mellitus
}

\author{
Authors \\ Aleksandra Klisic ${ }^{1}$, Aleksandra Isakovic ${ }^{2}$, Gordana Kocic ${ }^{3}$, Nebojsa Kavaric ${ }^{1}$, Milovan Jovanovic ${ }^{1}$, Elvir Zvrko ${ }^{4}$, \\ Verica Skerovic ${ }^{4}$, Ana Ninic 5
}

\author{
Affiliations \\ 1 Primary Health Care Center, Podgorica, Montenegro \\ 2 Institute of Medical and Clinical Biochemistry, University \\ of Belgrade - School of Medicine, Belgrade, Serbia \\ 3 Department of Medical Biochemistry, University of Nis \\ - School of Medicine, Nis, Serbia \\ 4 Clinical Center of Montenegro, Podgorica, Montenegro \\ 5 Department of Medical Biochemistry, University of \\ Belgrade - Faculty of Pharmacy, Belgrade, Serbia
}

\section{Key words}

dyslipidemia, fatty liver, inflammation, oxidative stress, type 2 diabetes

$\begin{array}{ll}\text { received } & 02.06 .2017 \\ \text { revised } & 18.07 .2017 \\ \text { accepted } & 21.08 .2017\end{array}$

\section{Bibliography}

DOI https://doi.org/10.1055/s-0043-118667

Published online: 11.9.2017

Exp Clin Endocrinol Diabetes 2018; 126: 371-378

(c) J. A. Barth Verlag in Georg Thieme Verlag KG Stuttgart .

New York

ISSN 0947-7349

\author{
Correspondence \\ Aleksandra Klisic, MD, PhD \\ Center of Laboratory Diagnostics \\ Primary Health Care Center \\ Trg Nikole Kovacevica 6 \\ 81000 Podgorica \\ Montenegro \\ Tel.: + 382/20/481 999, Fax: + 382/20/481999 \\ aleksandranklisic@gmail.com
}

\begin{abstract}
Introduction/Aim Considering the high prevalence of nonalcoholic fatty liver disease (NAFLD) in individuals with type 2 diabetes mellitus (DM2), we aimed to investigate the potential benefit of determining markers of oxidative stress, inflammation and dyslipidemia for prediction of NAFLD, as estimated with fatty liver index (FLI) in individuals with DM2.

Methods A total of 139 individuals with DM2 (of them 49.9\% females) were enrolled in cross-sectional study. Anthropometric and biochemical parameters, as well as blood pressure were obtained. A FLI was calculated.

Results Multivariate logistic regression analysis showed that high density lipoprotein cholesterol (HDL-c) and malondialdehyde (MDA) were independent predictors of higher FLI [Odds ratio $(O R)=0.056, p=0.029$; and $O R=1.105, p=0.016$, respectively]. In Receiver Operating Characteristic curve analysis, the addition of fatty liver risk factors (e. g., age, gender, body height, smoking status, diabetes duration and drugs metabolized in liver) to each analysed biochemical parameter [HDL-c, non-HDL-c, high sensitivity C-reactive protein (hsCRP), MDA and advanced oxidant protein products (AOPP)] in Model 1, increased the ability to discriminate patients with and without fatty liver [Area under the curve $(\mathrm{AUC})=0.832$, AUC $=0.808$, $\mathrm{AUC}=0.798, \mathrm{AUC}=0.824$ and $\mathrm{AUC}=0.743$, respectively $]$. Model 2 (which included all five examined predictors, e. g., HDL-c, non-HDL-c, hsCRP, MDA, AOPP, and fatty liver risk factors) improved discriminative abilities for fatty liver status ( $A \cup C=0.909$ ). Even more, Model 2 had the highest sensitivity and specificity ( $89.3 \%$ and $87.5 \%$, respectively) together than each predictor in Model 1.

Conclusion Multimarker approach, including biomarkers of oxidative stress, dyslipidemia and inflammation, could be of benefit in identifying patients with diabetes being at high risk of fatty liver disease.
\end{abstract}

\section{Introduction}

Non-alcoholic fatty liver disease (NAFLD) is the commonest form of chronic liver diseases [1] and is tightly associated with insulin resistance [2,3]. Therefore, the high prevalence of this metabolic disorder observed in individuals with obesity or type 2 diabetes mel- litus (DM2), [4] is not suprising. It is estimated that more than $60-70 \%$ of DM2 patients are affected by NAFLD $[4,5]$.

There is also evidence of association between NAFLD and longterm diabetes complications such as diabetic retinopathy and chronic kidney disease [6]. 
In addition, diabetic individuals with NAFLD have 2 fold increase risk for development and progression of cardiovascular disease [7], and also 2 to 3 fold higher risk of dying of chronic liver disease [8], compared to diabetic individuals without NAFLD.

The pathophysiological mechanism underlying NAFLD is not well elucidated. However, it is speculated that oxidative stress and inflammation are the key determinants of this hepatic manifestation of metabolic syndrome [9-11]. Namely, increased visceral adipose tissue is significant source of reactive oxygen species (ROS), higher pro-inflammatory adipokines and citokines [12,13], along with decreased antioxidant enzymatic [14] and non-enzymatic defence $[15,16]$, which altogether make the milleu of increased inflammation and oxidative stress [15, 17]. Furthermore, ROS impact insulin signalling pathways, thus leading to consequent insulin resistant state, increased free fatty acids hepatic influx, increased lipogenesis, as well as triglyceride storage, inducing hepatocytes dysfunction or death [18].

To our knowledge, there are no data examining the oxidative stress markers in relation to NAFLD in exclusively patients with DM2. Furthermore, considering the high prevalence of NAFLD in individuals with DM2, and its association with diabetes complications, we aimed to investigate the potential benefit of determining markers of lipids oxidative damage [e. g., malondialdehyde (MDA)], proteins oxidative damage [e.g., advanced oxidant protein products (AOPP)], as well as antioxydant enzyme [e. g., catalase (CAT)], inflammation and dyslipidemia for improvement the prediction of NAFLD, as estimated with fatty liver index (FLI) in a cohort of individuals with DM2.

\section{Subjects and Methods}

\section{Study population}

This cross-sectional study derived from a previous work aiming to evaluate the utility of visceral adiposity indexes in individuals with DM2 [19].

The study enrolled a total of 139 sedentary DM2 (of them $49.9 \%$ females) who volunteered to participate in the study. All examined patients were recruited by the endocrinologist in the Center of Laboratory Diagnostics of the Primary Health Care Center in Podgorica, Montenegro, for their regular biochemical analyses check-up in a period from October 2015 to May 2016. Medical history and clinical examinations were carried out on the same day.

Out of the total number of 362 patients with diabetes (207 men and 155 women), who were screened for the study, 139 of them met the criteria for inclusion in the study (71 men and 68 women).

The methods and assays used to include participants and to exclude disorders in participants with diabetes have been described in detail elsewhere [19].

Inclusion criteria for participation in the study were: sedentary patients ( $<90$ min of weekly exercise) with DM2. Diabetes cases were defined as self-reported diabetes, or with at least two elevated plasma glucose levels (fasting glucose $\geq 7.0 \mathrm{mmol} / \mathrm{L}$, a random plasma glucose level of $\geq 11.1 \mathrm{mmol} / \mathrm{L}$, or a plasma glucose level $\geq 11.1 \mathrm{mmol} / \mathrm{L} 2 \mathrm{~h}$ after an oral glucose tolerance test), or HBA1c $\geq 6.5 \%$ on two different occasions in the absence of symp- toms; or treatment with antidiabetic medication (insulin or oral antihyperglycemic agents) [19].

Exclusion criteria were: type 1 diabetes mellitus, liver disease other than NAFLD, ethanol consumption $>20 \mathrm{~g} /$ day, acute inflammatory disease, high sensitivity C-reactive protein levels (hsCRP) $>10 \mathrm{mg} / \mathrm{L}$, pregnancy, history or the presence of malignancy, as well as participants who were unwilling to enter the study [19].

Non-alcoholic fatty liver disease is assessed by FLI, as described previously $[2,20]$. A FLI score $\geq 60$ has been shown to have good specificity (80.3\%) and sensitivity (87.3\%) for established NAFLD, as reported previously $[5,21]$.

Although a $\mathrm{FLI}<30$ is regarded to exclude fatty liver, and that $\mathrm{FLI} \geq 60$ is suggestive of fatty liver, we have excluded participants with $30 \leq \mathrm{FLI}<60(n=41)$. Therefore, all participants that were eligible to enter the study were divided into two groups $(\mathrm{FLI}<30$, $\mathrm{n}=17$; and $\mathrm{FLI} \geq 60 ; \mathrm{n}=122$ ).

A total of $87.1 \%$ of participants used oral antihyperglycemics [of them metformin, sulfonylureas, inhibitors of dipeptidyl peptidase 4 (DPP-4 inhibitors) were used by $81.8 \%, 5.8 \%$, and $17.1 \%$ patients, respectively], whereas $16.5 \%$ of them were on insulin therapy. All participants who used lipid-modifying drugs (46.8\%) in our study used statins ( $100 \%$ of them), whereas the smaller number of them used fibrates, also (4.6\%). Antihypertensive medication usage was recorded in $73.4 \%$ participants [of them angiotensin converting enzyme inhibitors (ACE inhibitors), beta-blockers, and diuretics were used by $87.3 \%, 20.6 \%$ and $52.9 \%$ patients, respectively].

All the participants provided written informed consent. The study protocol was approved by the Ethical Committee of Primary Health Care Center in Podgorica, Montenegro and the research was carried out in compliance with the Declaration of Helsinki.

\section{Anthropometric measurements}

Basic anthropometric measurements: body height $(\mathrm{cm})$, body weight $(\mathrm{kg})$ and waist circumference $(\mathrm{WC})(\mathrm{cm})$ were obtained, and body mass index (BMI) was calculated, as described previously $[2,19]$. Waist-to-height ratio (WHtR) was calculated as waist $(\mathrm{cm})$ divided by height $(\mathrm{cm})$.

\section{Biochemical analyses}

Biochemical parameters were measured as previously described [19]. The blood samples were taken between 7-9 h a.m., after $12-14 \mathrm{~h}$ of an overnight fast. Samples were left to clot for $30 \mathrm{~min}$ and then centrifuged at $3000 \mathrm{rpm}$ for $10 \mathrm{~min}$.

Glucose levels were determined immediately after the blood was drawn, whereas serum samples used for other analyses were divided into aliquots and stored at $-80^{\circ} \mathrm{C}$ before analyses. A whole blood in $\mathrm{K}_{2}$ EDTA was used for determination of HbA1c, and it was measured with immunoturbidimetric assay (Roche Cobas 400, Mannheim, Germany).

Serum levels of glucose, total cholesterol (TC), high density lipoprotein cholesterol (HDL-c), low density lipoprotein cholesterol (LDL-C), triglycerides (TG), uric acid, bilirubin, aspartat aminotransferase (AST), alanine aminotransferase (ALT) and gamma-glutamil transferase (GGT), were measured as described previously [19], using standardized enzymatic procedure (Roche Cobas 400, Mannheim, Germany). High sensitivity C-reactive protein (hsCRP) lev- 
els were determined using a nephelometric assay (Behring Nephelometer Analyzer, Marburg, Germany).

Determination of serum AOPP was based on spectrophotometric detection of chloramine-T equivalents. In order to minimize the impact of storage time of samples as well as the possible influence of triglycerides and turbidity of samples, we modified AOPP assay by precipitating VLDL and LDL in the plasma. It was reported in a number of clinical disease states and method was developed in our previous reports [22].

Serum MDA levels were measured spectophotometrically. The MDA determination by measuring TBARS by thiobarbituric acid (TBA) test is described as the most-commonly employed approach. It was reported as a true measure of MDA in a number of clinical disease states and in our previous reports [23].

Catalase (CAT) test is based on the release of oxygen from hydrogen peroxide $\left(\mathrm{H}_{2} \mathrm{O}_{2}\right)$, by using the spectrophotometric assay based on formation of its stable complex with ammonium molybdate. Catalase activity determination was reported in a number of clinical disease states and method was developed in our previous reports. Lipemic plasma was without influence on the CAT assay [23].

Blood pressure was measured and Glomerular filtration rate was estimated by using creatinine in the Modification of Diet in Renal Disease Study equation (eGFR $\mathrm{MDRD}_{\text {) }}$ ) as described previously [19].

\section{Statistical analysis}

Data were presented as median (interquartile range) and compared by Mann-Whitney U-test. Comparison of categorical data given as absolute frequencies was performed with Chi-square test. Spearman's correlation analysis was used to estimate the correlation between clinical parameters and FLI. Logistic regression analysis with enter selection principle was used to analyze determination of one or more independent or predictor variables (demographic characteristics, inflammation, lipid and oxidative stress markers) on the occurrence of fatty liver (dichotomous dependent variable). Also, it was used to identify independent determinants of FLI. Linear relationship between continuous predictor variables and the logit transformation of the dependent variable (FLI) was confirmed by Hosmer-Lemeshow test. Data are given as odds ratio (OR) and $95 \%$ confidence interval $(\mathrm{Cl})$ for odds. The explained variation in dependent variable was presented by Nagelkerke R Squared (pseudo $R^{2}$ ) value. The diagnostic potential of predictors was evaluated using Receiver Operating Characteristic (ROC) curve analysis. The area under ROC curve (AUC) was used as a measure how well predictors could distinguish between subjects that suffered from fatty liver from those who did not. All statistical calculations were performed using PASW $®$ Statistic version 18 (Chicago, Illinois, USA) and the MedCalc ${ }^{\circledR}$ (Mariakerke, Belgium) Version 15.8. Two-tailed $p$ values less than 0.05 were considered as statistically significant.

\section{Results}

The general characteristics of patients with DM2 divided according to the FLI values are indicated in $>$ Table 1. As expected, there were significant differences between groups in all parameters (except for height) which were used in FLI calculation. Body weight, BMI, WC, WHtR were statistically higher in the group with $\mathrm{FLI} \geq 60$ than in the group with FLI $<30$ ( $p<0.001$ for all). Also, patients in the group with $\mathrm{FLI} \geq 60$ had significantly higher SBP than those in the group with $\mathrm{FLI}<30(\mathrm{p}=0.027)$. Duration of diabetes of patients in the group with $\mathrm{FLI} \geq 60$ was significantly shorter than in the group with FLI $<30(p=0.010)$. There were unequal distributions of patients with smoking habits, hypolipemic and antihyperglycemic therapies usages according to Chi-square test ( $\triangleright$ Table 1 ).

A significantly lower HDL-c concentration $(p=0.003)$ was found in the group with $\mathrm{FLI} \geq 60$ than in the group with $\mathrm{FLI}<30$ ( $\vee$ Table 2 ). In contrast, higher TG concentration $(p<0.001)$ and high calculated indexes (e. g., TG/HDL-c ratio and non-HDL-c; $p<0.001$ and $p=0.027$, respectively) were evident in the group with $F L I \geq 60$. These results were not unexpected, because TG concentration entered equation for FLI calculation. We also demonstrated significantly higher levels of glucose, hsCRP, uric acid, MDA and AOPP $(p=0.038, p=0.002, p=0.033, p=0.001$ and $p=0.005$, respectively) in the group with $\mathrm{FLI} \geq 60$ comparing with the group with $\mathrm{FLI}<30$ ( $\triangleright$ Table 2).

Spearman's correlation analysis was used to test possible associations between FLI and examined clinical parameters in the cohort of patients with DM2 ( $>$ Table 3 ). We found a significant negative correlation between FLI and HDL-c $(p<0.001)$, FLI and years of age $(p=0.001)$ and FLI and diabetes duration $(p=0.013)$. Positive correlations were evident between FLI and concentrations of glucose, HBA1c, hsCRP, MDA and AOPP ( $p=0.001, p=0.009$, $p<0.001, p=0.015$ and $p=0.019$, respectively).

Multivariate logistic regression analysis was used to further investigate the associations of clinical parameters which were significantly different between the group with FLI $\geq 60$, and the group with $\mathrm{FLI}<30$, with fatty liver development, unadjusted and after adjustment for other risk factors ( $\triangleright$ Table 4). Unadjusted ORs showed that HDL-c, non-HDL-c, hsCRP, uric acid, MDA and AOPP had significant potential for fatty liver risk prediction. Also, unadjusted regression models correctly classified around $88 \%$ of patients in the group having fatty liver. Model 1 presented adjustment for fatty liver risk factors (e. g., age, gender, body height, smoking status, diabetes duration and drugs metabolized in liver) with HDLc, non-HDL-c, hsCRP, MDA and AOPP respectively, and revealed that HDL-c, non-HDL-c, hsCRP and MDA were the independent risk predictors for fatty liver occurrence. As HDL-c concentration fall for $1 \mathrm{mmol} / \mathrm{L}$, probability for higher fatty liver occurrence risk rose for $97.6 \%(O R=0.024, p=0.001)$. As non-HDL-c rose for $1 \mathrm{mmol} / \mathrm{L}$, probability for fatty liver occurence rose almost 3 times $(O R=2.725$, $\mathrm{p}=0.006$ ). With increment in hsCRP concentration for $1 \mathrm{mg} / \mathrm{L}$, probability for higher fatty liver occurrence risk rose for $37.9 \%$ $(O R=1.379, p=0.043)$. Furthermore, with increment in MDA concentration for $1 \mu \mathrm{mol} / \mathrm{L}$, probability for higher fatty liver occurrence risk rose for $12.0 \%(O R=1.120, p=0.001)$, (

In Model 2, the adjustment was performed for fatty liver risk factors (e. g., age, gender, body height, smoking status, diabetes duration, drugs metabolized in liver), together with HDL-c, non-HDLc, hsCRP, MDA and AOPP. Results for Model 2 showed that HDL-c and MDA still kept significant prediction potential for fatty liver development $(O R=0.056, p=0.029$; and $O R=1.105, p=0.016$, respectively). Also, $50.8 \%$ of variation (given as Nagelkerke $R^{2}$ in - Table 4) in fatty liver development could be explained by the Model 2. Furthermore, according to the Model 2, a total of $91.3 \%$ of patients could be correctly classified in the group having fatty liver. 
- Table 1 Demographic characteristics of patients with diabetes according to FLI.

\begin{tabular}{|c|c|c|c|}
\hline & FLI $<30 \mathrm{~N}=17(12.2 \%)$ & FLI $\geq 60 \mathrm{~N}=122(87.8 \%)$ & $\mathbf{p}$ \\
\hline $\mathrm{N}$ (males/females) & $17(9 / 8)$ & $122(62 / 60)$ & 0.683 \\
\hline Age, years & $63(53-70)$ & $63(55-69)$ & 0.788 \\
\hline Body weight, kg & $72(69-79)$ & $93(84-100)$ & $<0.001$ \\
\hline Body height, $\mathrm{cm}$ & $173(168-177)$ & $172(165-179)$ & 0.641 \\
\hline $\mathrm{BMI}, \mathrm{kg} / \mathrm{m}^{2}$ & $25.2(23.3-25.9)$ & $31.1(28.7-34.1)$ & $<0.001$ \\
\hline WC, $\mathrm{cm}$ & $90(86-92)$ & $110(104-117)$ & $<0.001$ \\
\hline WHtR & $0.51(0.50-0.54)$ & $0.64(0.60-0.69)$ & $<0.001$ \\
\hline $\mathrm{SBP}, \mathrm{mmHg}$ & $129(136-144)$ & $135(126-144)$ & 0.028 \\
\hline $\mathrm{DBP}, \mathrm{mmHg}$ & $70(66-82)$ & $80(74-85)$ & 0.064 \\
\hline Smoking habits, (Smokers/Non-smokers) & $4 / 13$ & $28 / 94$ & 0.011 \\
\hline $\begin{array}{l}\text { Antihyperglycemics (Yes/No) } \\
\text {-Metformin }(\mathrm{N}=99) \\
\text {-Sulfonylurea }(\mathrm{N}=7) \\
\text {-DPP-4 inhibitors }(\mathrm{N}=22)\end{array}$ & $15 / 2$ & $106 / 16$ & 0.028 \\
\hline Insulin (Yes/No) & $5 / 12$ & $18 / 104$ & 0.11 \\
\hline $\begin{array}{l}\text { Hypolipemics (Yes/No) } \\
\text {-Statins }(\mathrm{N}=65) \\
\text {-Fibrates }(\mathrm{N}=3)\end{array}$ & $9 / 8$ & $56 / 66$ & 0.009 \\
\hline $\begin{array}{l}\text { Antihypertensives (Yes/No) } \\
\text {-ACE inhibitors }(\mathrm{N}=89) \\
\text {-Beta blockers }(\mathrm{N}=21) \\
\text {-Diuretics }(\mathrm{N}=54)\end{array}$ & $9 / 8$ & $93 / 29$ & 0.119 \\
\hline Duration of diabetes, years & $7.0(3.5-10.0)$ & $3.50(1.0-7.0)$ & 0.01 \\
\hline
\end{tabular}

- Table 2 Clinical parameters in patients with diabetes according to FLI.

\begin{tabular}{|c|c|c|c|}
\hline & FLI $<30 \mathrm{~N}=17(12.2 \%)$ & $\mathrm{FLI} \geq 60 \mathrm{~N}=122(87.8 \%)$ & $\mathbf{p}$ \\
\hline $\mathrm{TC}, \mathrm{mmol} / \mathrm{L}$ & $4.79(4.19-5.78)$ & $5.25(4.57-6.02)$ & 0.135 \\
\hline $\mathrm{HDL}-\mathrm{c}, \mathrm{mmol} / \mathrm{L}$ & $1.54(1.01-1.63)$ & $1.10(0.88-1.32)$ & 0.003 \\
\hline $\mathrm{LDL}-\mathrm{c}, \mathrm{mmol} / \mathrm{L}$ & $3.15(2.34-3.50)$ & $3.07(2.49-3.89)$ & 0.528 \\
\hline $\mathrm{TG}, \mathrm{mmol} / \mathrm{L}$ & $1.12(0.86-1.43)$ & $2.22(1.68-3.17)$ & $<0.001$ \\
\hline TG/HDL-c ratio & $0.86(0.59-1.04)$ & $2.11(1.28-2.98)$ & $<0.001$ \\
\hline Non-HDL-c & $3.66(2.75-4.21)$ & $4.08(3.48-5.09)$ & 0.025 \\
\hline Glucose, mmol/L & $6.50(5.65-8.55)$ & $7.45(6.50-9.10)$ & 0.038 \\
\hline HBA1c, \% & $5.85(5.55-7.90)$ & $6.75(6.00-8.00)$ & 0.148 \\
\hline AST, U/L & $17(14-18)$ & $20(17-24)$ & 0.003 \\
\hline ALT, U/L & $16(14-19)$ & $24(17-35)$ & 0.001 \\
\hline GGT, U/L & $11(10-19)$ & $24(17-34)$ & $<0.001$ \\
\hline $\mathrm{HsCRP}, \mathrm{mg} / \mathrm{L}$ & $0.73(0.45-1.85)$ & $1.75(1.06-5.15)$ & 0.002 \\
\hline Total bilirubin, $\mu \mathrm{mol} / \mathrm{L}$ & $7.20(3.85-12.90)$ & $6.00(4.80-8.30)$ & 0.641 \\
\hline Creatinine, $\mu \mathrm{mol} / \mathrm{L}$ & $67(58-83)$ & $73(63-86)$ & 0.367 \\
\hline Uric acid, $\mu \mathrm{mol} / \mathrm{L}$ & $259(204-339)$ & $321(265-360)$ & 0.033 \\
\hline $\mathrm{eGFR}_{\mathrm{MDRD}}, \mathrm{mL} / \mathrm{min} / 1.73 \mathrm{~m}^{2}$ & $90(81-95)$ & $81(70-101)$ & 0.301 \\
\hline $\mathrm{MDA}, \mu \mathrm{mol} / \mathrm{L}$ & $44.45(36.47-54.04)$ & $55.19(47.80-63.31)$ & 0.001 \\
\hline AOPP, $\mathrm{T} / \mathrm{L}$ & $58.66(50.25-100.36)$ & $81.88(67.50-121.66)$ & 0.005 \\
\hline CAT, U/L & $70.90(57.08-93.10)$ & $70.59(32.88-110.80)$ & 0.855 \\
\hline
\end{tabular}


- Table 3 Associations between FLI and clinical parameters using Spearman's correlation analysis in patients with diabetes.

\begin{tabular}{|l|c|c|}
\hline Variable & Rho $(\boldsymbol{\rho})$ & $\mathbf{p}$ \\
\hline Age, years & -0.277 & 0.001 \\
\hline Body height, cm & -0.075 & 0.385 \\
\hline Diabetes duration, years & -0.211 & 0.013 \\
\hline SBP, mm Hg & 0.260 & 0.002 \\
\hline DBP, $\mathrm{mm} \mathrm{Hg}$ & 0.195 & 0.022 \\
\hline TC, mmol/L & 0.048 & 0.578 \\
\hline HDL-c, mmol/L & -0.358 & $<0.001$ \\
\hline LDL-c, mmol/L & -0.008 & 0.927 \\
\hline Non-HDL-c, mmol/L & 0.114 & 0.099 \\
\hline Glucose, mmol/L & 0.268 & 0.001 \\
\hline HBA1c, \% & 0.222 & 0.009 \\
\hline HsCRP, mg/L & 0.359 & $<0.001$ \\
\hline Uric acid, $\mu \mathrm{mol} / \mathrm{L}$ & 0.123 & 0.151 \\
\hline Creatinine, $\mu \mathrm{mol} / \mathrm{L}$ & -0.072 & 0.402 \\
\hline eGFR $\mathrm{MDRD}, \mathrm{mL} / \mathrm{min} / 1.73 \mathrm{~m}^{2}$ & 0.139 & 0.105 \\
\hline MDA, $\mu \mathrm{mol} / \mathrm{L}$ & 0.208 & 0.015 \\
\hline AOPP, T/L & 0.199 & 0.019 \\
\hline CAT, U/L & 0.100 & 0.245 \\
\hline Data age given as coefficients of correlation Rho $(\rho)$ & \\
\hline & & \\
\hline
\end{tabular}

The aim of our study was to investigate the potential benefit of determining clinical parameters which were different between FLI groups in order to discriminate patients with DM2 with fatty liver disease from those who did not have it. ROC curves were used to achieve this ( $\triangleright$ Table 5). The calculated AUCs for the measurement of single clinical parameter (from 0.660 to 0.753 ) indicated that the clinical accuracy of the applied procedures was low. The same models were used in ROC analysis as in multivariate logistic analysis. The addition of fatty liver risk factors in Model 1 beside each clinical parameter (HDL-C, non-HDL-c, hsCRP, MDA and AOPP) increased the ability to discriminate patients with diabetes with and without fatty liver $(A \cup C=0.832, A \cup C=0.808, A \cup C=0.798, A \cup C=0.824$ and $A \cup C=0.743$, respectively), ( $\vee$ Table 5, $\triangleright$ Fig. 1). Model 2 improved discriminative abilities for fatty liver development. Calculated AUC was 0.909 , which gave excellent accuracy of the applied procedure ( $>$ Table 5, > Fig. 2). Even more, the applied Model 2 had the highest sensitivity and specificity ( $89.3 \%$ and $87.5 \%$, respectively) together than each predictor in Model 1, ( $\vee$ Table 5).

\section{Discussion}

In the current study we reported high prevalence of FLI-NAFLD, accounting for $87.8 \%$ of participants with DM2 to have this metabolic disorder. Moreover, we observed an inverse association between age and FLI-NAFLD. The inverse association of FLI-NAFLD with age in our study differentiate this diabetic complication from established vascular complications, typically related to the duration of hyperglycemia [5].

Also, participants with $\mathrm{FLI}<30$ displayed longer duration of diabetes ( $\triangleright$ Table 1). Our results are in line with Giorda et al. [5] who showed in a large study comprising of more than 5,000 participants with diabetes, high prevalence of FLI-NAFLD (e. g., $61.3 \%$ ) which was more frequent among younger male patients or those with a shorter duration of diabetes. This unexpected results may be explained in part by diabetes treatment which may lower intrahepatic lipid content in the group with FLI <30, which appear healthier despite longer DM2 duration.

To our knowledge, this is the first study examining the oxidative stress markers in relation to FLI-NAFLD in exclusively patients with DM2. We previously reported insulin resistance, higher inflammation (as measured with hsCRP), and increased adipokine level such as retinol-binding protein 4 , as independent predictors of FLINAFLD in a cohort of postmenopausal, otherwise healthy women [2]. The current study extends those observations, suggesting that oxidative stress has independent influence on fatty liver development in patients with $\mathrm{DM} 2$.

Mitochondria and the endoplasmic reticulum of hepatocytes via the cytochrome P450 enzymes are the primary source of ROS, thus further leading to hepatic structural and functional disorders [24].

AOPPs indicates the overall status of the proteins in the cell/tissue, and in the states of increased oxidative stress they are created by reactions between plasma proteins and chlorinated oxidants [25].

Previous reports indicate that proteins are equally targeted by ROS as the lipids in diabetes [17], showing positive association between plasma levels of AOPPs and lipid peroxidation products. However, in the current study, after adjustment for confounding factors $[4,5,26]$ we revealed that higher MDA, but not AOPP level was the independent risk predictor for fatty liver occurrence. This finding suggests that MDA is superior to AOPP in fatty liver risk prediction. Namely, even though that both MDA and AOPP significantly correlated with FLI-NAFLD in our study ( $\vee$ Table 3 ), and although higher AOPP levels were recorded in the group with FLI $\geq 60$ ( $\triangleright$ Table 2), AOPPs were not retained in multivariate logistic regression analysis as independent predictor of fatty liver occurrence $(O R=1.014$, $\mathrm{p}=0.240$; Table 4). Nevertheless, in unadjusted model AOPP showed significant potential for fatty liver risk prediction.

Malondialdehyde (MDA) is reported to be a primary biomarker of lipid peroxidation of poly-unsaturated fatty acids that were attacked by ROS $[25,27]$ inducing multiple cellular alterations, influencing on the activity of mitochondrial respiratory chain, and generating more ROS, thus further increasing oxidative stress in NAFLD [18] and making a vitious circle between oxidative stress and NAFLD.

Several previous studies also reported higher level of oxidative damage markers, such as MDA, in individuals with NAFLD [28-30].

On the other hand, no significant difference between groups in CAT activity was reported ( $\vee$ Table 2 ), although previous studies reported decreased CAT activity $[28,29]$ in the plasma of individuals with NAFLD.

Antioxidant enzyme CAT converts hydrogen peroxide into oxygen and water and thus neutralizes it, since hydrogen peroxide is a highly reactive molecule formed as a natural by-product of energy metabolism and may cause, like other ROS significant damages to proteins, lipids, and DNA [25].

No difference in CAT activity in low vs. high FLI-NAFLD risk group in our study may be explained by the assumption that liver tissue tries to counteract oxidative stress induced by elevated free fatty acids influx, by increasing expression and activity of antioxidant enzymes, even though they are shown to be progressively depleted as metabolic disorder occurs [17]. 
- Table 4 Estimated odds ratios (OR) after multivariate logistic regression analysis for parameters predicting abilities regarding FLI.

\begin{tabular}{|c|c|c|c|c|}
\hline Predictors & Unadjusted OR (95\%Cl) & $\mathbf{p}$ & Nagelkerke $R^{2}$ & $\%$ of cases correctly classified \\
\hline HDL-c & $0.069(0.012-0.383)$ & 0.002 & 0.141 & 87.7 \\
\hline Non-HDL-c & $2.115(1.180-3.791)$ & 0.012 & 0.109 & 88.4 \\
\hline HsCRP & $1.372(0.993-1.895)$ & 0.055 & 0.086 & 88.4 \\
\hline Glucose & $1.291(0.961-1.736)$ & 0.090 & 0.058 & 88.4 \\
\hline Uric acid & $1.008(1.001-1.015)$ & 0.019 & 0.083 & 88.4 \\
\hline MDA & $1.100(1.039-1.165)$ & 0.001 & 0.180 & 89.1 \\
\hline AOPP & $1.023(1.002-1.045)$ & 0.035 & 0.077 & 88.4 \\
\hline \multicolumn{5}{|c|}{ Model 1} \\
\hline HDL-C & $0.024(0.003-0.203)$ & 0.001 & 0.300 & 89.9 \\
\hline Non-HDL-c & $2.725(1.334-5.565)$ & 0.006 & 0.261 & 87.7 \\
\hline HsCRP & $1.379(1.010-1.881)$ & 0.043 & 0.213 & 88.4 \\
\hline MDA & $1.120(1.048-1.196)$ & 0.001 & 0.322 & 89.9 \\
\hline AOPP & $1.022(1.002-1.045)$ & 0.068 & 0.179 & 88.4 \\
\hline \multicolumn{5}{|c|}{ Model 2} \\
\hline HDL-C & $0.056(0.004-0.746)$ & 0.029 & \multirow{5}{*}{0.508} & \multirow{5}{*}{91.3} \\
\hline Non-HDL-c & $1.961(0.776-4.951)$ & 0.154 & & \\
\hline HsCRP & $1.174(0.868-1.587)$ & 0.297 & & \\
\hline MDA & $1.105(1.019-1.198)$ & 0.016 & & \\
\hline AOPP & $1.014(0.991-1.039)$ & 0.240 & & \\
\hline \multicolumn{5}{|c|}{$\begin{array}{l}\text { Model 1: adjustment for age, body height, diabetes duration (all continuous variables), gender, smoking habits, therapies (all categorical variables) } \\
\text { and each predictor }\end{array}$} \\
\hline \multicolumn{5}{|c|}{$\begin{array}{l}\text { Model 2: adjustment for age, body height, diabetes duration (all continuous variables), gender, smoking habits, therapies (all categorical variables) } \\
\text { and all predictors }\end{array}$} \\
\hline \multicolumn{5}{|c|}{ CI-Confidence interval; SE-Standard error } \\
\hline
\end{tabular}

- Table 5 ROC analysis for single parameter and models discriminatory abilities regarding FLI.

\begin{tabular}{|c|c|c|c|c|c|}
\hline Predictors & AUC $(95 \% \mathrm{Cl})$ & SE & Sensitivity (\%) & Specificity (\%) & $\mathbf{p}$ \\
\hline HDL-C & $0.731(0.604-0.771)$ & 0.079 & 86.1 & 68.7 & 0.005 \\
\hline Non-HDL-c & $0.670(0.540-0.801)$ & 0.066 & 31.1 & 100.0 & 0.027 \\
\hline $\mathrm{HsCRP}$ & $0.744(0.599-0.890)$ & 0.074 & 80.3 & 68.7 & 0.002 \\
\hline Glucose & $0.660(0.507-0.813)$ & 0.078 & 73.0 & 56.2 & 0.038 \\
\hline Uric acid & $0.665(0.515-0.814)$ & 0.076 & 68.9 & 62.5 & 0.033 \\
\hline MDA & $0.753(0.636-0.870)$ & 0.060 & 84.4 & 56.2 & 0.001 \\
\hline AOPP & $0.715(0.632-0.788)$ & 0.085 & 85.2 & 56.2 & 0.012 \\
\hline \multicolumn{6}{|c|}{ Model 1} \\
\hline HDL-C & $0.832(0.727-0.937)$ & 0.054 & 84.4 & 75 & $<0.001$ \\
\hline Non-HDL-c & $0.808(0.689-0.927)$ & 0.061 & 90.2 & 62.5 & $<0.001$ \\
\hline $\mathrm{HsCRP}$ & $0.798(0.674-0.921)$ & 0.063 & 75.4 & 81.2 & $<0.001$ \\
\hline MDA & $0.824(0.716-0.932)$ & 0.055 & 86.1 & 68.7 & $<0.001$ \\
\hline AOPP & $0.743(0.604-0.882)$ & 0.071 & 81.1 & 62.5 & 0.002 \\
\hline \multicolumn{6}{|c|}{ Model 2} \\
\hline HDL-C & \multirow{5}{*}{$0.909(0.848-0.951)$} & \multirow{5}{*}{0.044} & \multirow{5}{*}{89.3} & \multirow{5}{*}{87.5} & \multirow{5}{*}{$<0.001$} \\
\hline Non-HDL-c & & & & & \\
\hline $\mathrm{HsCRP}$ & & & & & \\
\hline MDA & & & & & \\
\hline AOPP & & & & & \\
\hline \multicolumn{6}{|c|}{$\begin{array}{l}\text { Model 1: adjustment for age, body height, diabetes duration (all continuous variables), gender, smoking habits, therapies (all categorical variables) } \\
\text { and each predictor }\end{array}$} \\
\hline \multicolumn{6}{|c|}{$\begin{array}{l}\text { Model 2: adjustment for age, body height, diabetes duration (all continuous variables), gender, smoking habits, therapies (all categorical variables) } \\
\text { and all predictors }\end{array}$} \\
\hline
\end{tabular}




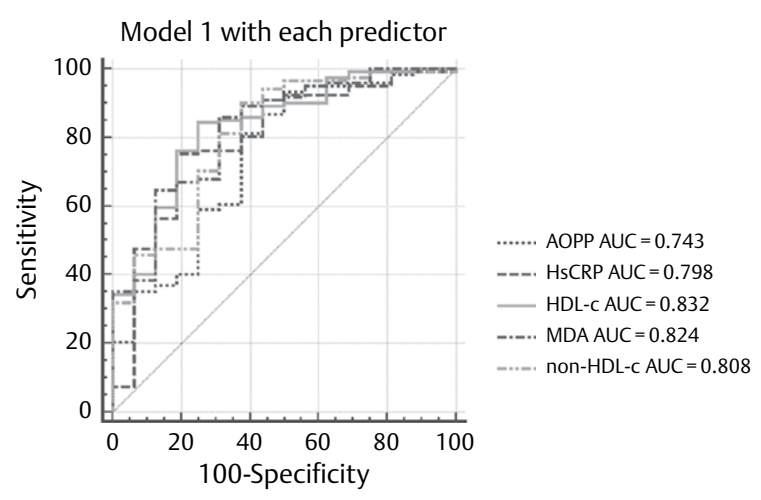

- Fig. 1 ROC curves for Model 1 (with each predictor) discriminative ability for fatty liver development.

In the state of visceral obesity, increased free fatty acids hepatic influx enhance oxidative phosphorylation, leading to increase ROS/RNS production and oxidative stress, but also promoting inflammation through enhanced macrophage infiltration and increased secretion of broad spectrum of pro-inflammatory adipoand cytokines, with consequent impairement of insulin action and dyslipidemia [12, 17, 18]. Inflammation in the adipose tissue may, therefore, precede hepatic inflammation [16]. In addition, secondary to insulin resistance, increased cellular uptake of free faty acids without any subsequent $\beta$-oxidation contributes to the increased triglicerides production that, in turn, stimulates secretion of very low density lipoprotein (VLDL) in hepatocytes, further contributing to hepatic steatosis exacerbation $[11,12]$.

In our study, after adjustment for all fatty liver risk factors $[4,5,26]$, HDL-c and MDA, still kept significant prediction potential for fatty liver ( $\triangleright$ Table 4 ).

Since the aim of our study was to investigate the potential benefit of determining markers of oxidative stress, inflammation and dyslipidemia in order to discriminate patients with DM2 with fatty liver disease from those who did not have it, we performed ROC analysis. It is important to note that Model 2 (which included confounding factors, and all five examined predictors, e. g., HDL-c, non-HDL-c, hsCRP, MDA, AOPP) improved discriminative ability for fatty liver development ( $A U C=0.909$ ). Even more, the applied procedure had the highest sensitivity and specificity $(89.3 \%$ and $87.5 \%$, respectively) together than each predictor in Model 1 ( Table 5), suggesting that multifactorial approach including oxidative stress markers, inflammation markers, and markers of dyslipidemia, could be of great benefit in discriminating patients with DM2 with FLI-NAFLD from those individuals with DM2, but without FLI-NAFLD. Also, high sensitivity of the applied procedure (nearly $90 \%$ ) could be used as a good screening procedure in order not to miss any patient having fatty liver disease and to detect this disorder as it really exists.

In addition, a very recent study proposed the extended FLI which significantly improves the power of the FLI to predict NAFLD [31]. Namely, the authors demonstrated that the fold-change of plasma triglycerides during a $2 \mathrm{~h}$ oral glucose tolerance test and $2 \mathrm{~h}$ glucose

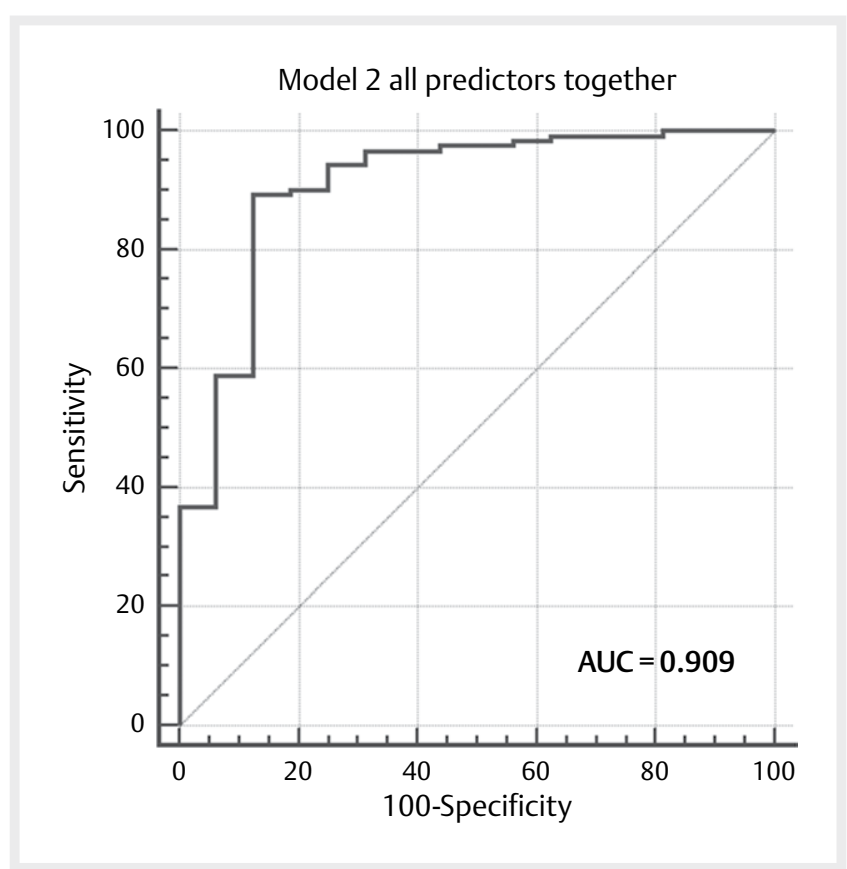

- Fig. 2 ROC curve for Model 2 discriminative ability for fatty liver development.

levels, together with the rs 738409 C $>$ G single nucleotide polymorphism in PNPLA3 may improve the power of the widely used FLI for NAFLD prediction.

The main disadvantages of the current study are the small sample size of our cohort and its cross-sectional design. Moreover, we have only calculated the FLI and had no direct measurements of hepatic steatosis, such as ultrasound, computed tomography, proton magnetic resonance spectroscopy or liver biopsy. Nevertheless, since FLI score $\geq 60$ has been shown to have a good sensitivity and specificity for established NAFLD [21], we suggest that multifactorial and multimarker approach, including biomarkers of oxidative stress and inflammation, in addition to markers od dyslipidemia could be of benefit in identifying patients with DM2, having a great risk of fatty liver disease. Further studies are needed to confirm our results.

\section{Conclusion}

Prevention and/or early recognizing of non-alcoholic fatty liver disease is of urgent need in patients with type 2 diabetes mellitus. In addidition to traditional risk factors for the onset and progression of fatty liver disease, multimarker approach including oxidative stress markers, markers of dyslipidemia, as well as inflammation markers could greatly improve the early identification of those patients with high risk of fatty liver disease and its consequences.

\section{Acknowledgement}

This work was financially supported in part by a grant from the Ministry of Education, Science and Technological Development, Republic of Serbia (Project number 175035). 


\section{Conflict of Interest}

The authors declared no conflicts of interest.

\section{References}

[1] Amirkalali B, Sohrabi MR, Esrafily A et al. Association between Nicotinamide Phosphoribosyltransferase and de novo Lipogenesis in Nonalcoholic Fatty Liver Disease. Med Princ Pract 2017; 26: 251-257

[2] Klisic A, Kavaric N, Jovanovic $\mathrm{M}$ et al. Bioavailable testosterone is independently associated with fatty liver index in postmenopausal women. Arch Med Sci 2017; 13: 1188-1196

[3] Macut D, Tziomalos K, Božić-Antić I et al. Non-alcoholic fatty liver disease is associated with insulin resistance and lipid accumulation productin women with polycystic ovary syndrome. Hum Reprod 2016; 31: 1347-1353

[4] Hazlehurst JM, Woods C, Marjot T et al. Non-alcoholic fatty liver disease and diabetes. Metabolism 2016; 65: 1096-1108

[5] Giorda C, Forlani G, Manti R et al. Occurrence over time and regression of nonalcholic fatty liver disease in type 2 diabetes. Diabetes Metab Res Rev 2017; e2878

[6] Targher G, Bertolini L, Chonchol M et al. Nonalcoholic fatty liver disease is independently associated with an increased prevalence of chronic kidney disease and retinopathy in type 1 diabetic patients. Diabetologia 2010; 53: 1341-1348

[7] Targher G, Bertolini L, Rodella S et al. Nonalcoholic fatty liver disease is independently associated with an increased incidence of cardiovascular events in type 2 diabetic patients. Diabetes Care 2007; 30: 2119-2121

[8] Zoppini G, Fedeli U, Gennaro N et al. Mortality from chronic liver diseases in diabetes. Am J Gastroenterol 2014; 109: 1020-1025

[9] Reyes-Gordillo K, Shah R, Muriel P. Oxidative stress and inflammation in hepatic diseases: Current and future therapy. Oxid Med Cell Longev 2017; 2017: 3140673

[10] de Andrade KQ, Moura FA, dos Santos JM et al Oxidative stress and inflammation in hepatic diseases: Therapeutic possibilities of n-acetylcysteine. Int J Mol Sci 2015; 16: 30269-30308

[11] Gaggini M, Morelli M, Buzzigoli E et al. Non-alcoholic fatty liver disease (NAFLD) and its connection with insulin resistance, dyslipidemia, atherosclerosis and coronary heart disease. Nutrients 2013; 5: 1544-1560

[12] Papaetis GS, Papakyriakou P, Panagiotou TN. Central obesity, type 2 diabetes and insulin: Exploring a pathway full of thorns. Arch Med Sci 2015; 11: 463-482

[13] Klisic AN, Vasiljevic ND, Simic TP et al. Association between C-reactive protein, anthropometric and lipid parameters among healthy normal weight and overweight postmenopausal women in Montenegro. Lab Med 2014; 45: 12-16

[14] Klisic A, Kotur-Stevuljevic ], Kavaric $\mathrm{N}$ et al. The association between follicle stimulating hormone and glutathione peroxidase activity is dependent on abdominal obesity in postmenopausal women. Eat Weight Disord - St 2016, doi:10.1007/s40519-016-0325-1
[15] Klisic A, Kavaric N, Soldatovic I et al. Relationship between cardiovascular risk score and traditional and nontraditional cardiometabolic parameters in obese adolescent girls. J Med Biochem 2016; 35: 282-292

[16] Ipsen DH, Tveden-Nyborg P, Lykkesfeldt L. Does vitamin c deficiency promote fatty liver disease development? Nutrients 2014; 6: 5473-5499

[17] Savini I, Catani MV, Evangelista D et al. Obesity-associated oxidative stress: strategies finalized to improve redox state. Int J Mol Sci 2013; 14 (5): 10497-10538

[18] Li S, Tan HY, Wang N et al. The role of oxidative stress and antioxidants in liver diseases. Int J Mol Sci 2015; 16: 26087-26124

[19] Kavaric N, Klisic A, Ninic A. Are visceral adiposity index and lipid accumulation product reliable indices for metabolic disturbances in patients with type 2 diabetes mellitus? J Clin Lab Anal 2017, doi: $10.1002 /$ jcla. 22283

[20] Bedogni G, Bellentani S, Miglioli L et al. The Fatty Liver Index: a simple and accurate predictor of hepatic steatosis in the general population. BMC Gastroenterol 2006; 6: 33

[21] Zelber-Sagi S, Webb M, Assy $\mathrm{N}$ et al. Comparison of fatty liver index with noninvasive methods for steatosis detection and quantification. World J Gastroenterol 2013; 19: 57-64

[22] Tasić D, Radenkovic S, Stojanovic D et al. Crosstalk of various biomarkers that might provide prompt identification of acute or chronic cardiorenal syndromes. Cardiorenal Med 2016; 6: 99-107

[23] Kocić R, Pavlović D, Kocić G. Impact of intensive insulin treatment on the development and consequences of oxidative stress in insulin dependent diabetes mellitus. Vojnosanit Pregl 2007; 64: 623-628

[24] Cichoż-Lach H, Michalak A. Oxidative stress as a crucial factor in liver diseases. World J Gastroenterol 2014; 20: 8082-8091

[25] Tiwari BK, Pandey KB, Abidi AB et al. Markers of oxidative stress during diabetes mellitus. J Biomark 2013; 2013: 378790.

[26] Stefan N, Häring HU, Hu FB et al. Divergent associations of height with cardiometabolic disease and cancer: Epidemiology, pathophysiology, and global implications. Lancet Diabetes Endocrinol 2016; 4: 457-467

[27] Lee SM, Cho YH, Lee SY et al. Urinary Malondialdehyde Is Associated with Visceral Abdominal Obesity in Middle-Aged Men. Mediators Inflamm 2015; 2015; 524291

[28] Kumar A, Sharma A, Duseja A et al. Patients with nonalcoholic fatty liver disease (NAFLD) have higher oxidative stress in comparison to chronic viral hepatitis. J Clin Exp Hepatol 2013; 3: 12-18

[29] Samy W, Hassanian MA. Paraoxonase-1 activity, malondialdehyde and glutathione peroxidase in non-alcoholic fatty liver disease and the effect of atorvastatin. Arab J Gastroenterol 2011; 12: 80-85

[30] Köroğlu E, Canbakan B, Atay K et al. Role of oxidative stress and insulin resistance in disease severity of non-alcoholic fatty liver disease. Turk J Gastroenterol 2016; 27: 361-366

[31] Kantartzis K, Rettig I, Staiger $\mathrm{H}$ et al. An extended fatty liver index to predict non-alcoholic fatty liver disease. Diabetes Metab 2017; 43: 229-239 